\title{
YEGUA QUEMADA 3: UN DEPÓSITO ARQUEOLÓGICO DEL HOLOCENO MEDIO EN EL PARQUE NACIONAL MONTE LEÓN (PATAGONIA, ARGENTINA)
}

MARÍA SOLEDAD CARACOTCHEa, A. SEBASTIÁN MUÑOZ DANIELA S. CAÑETE MASTRÁNGELO. ${ }^{c} \&$ PATRICIA A. LOBBIA ${ }^{b}$

\section{RESUMEN}

En este trabajo se presentan los resultados obtenidos en el sitio arqueológico Yegua Quemada 3 (YQ 3), Parque Nacional Monte León, Argentina. Se trata de un depósito estratigráfico ubicado a un metro y diez centímetros de profundidad en un acantilado de ocho metros de altura sobre la línea de costa actual y está datado en el Holoceno medio. Los recursos animales explotados incluyen mamíferos grandes y pequeños, aves, peces, e invertebrados e involucran una diversidad de técnicas de captura que permitieron el aprovechamiento de una variedad de recursos estáticos, móviles, terrestres y marinos. El material lítico recuperado está confeccionado tanto sobre materias primas locales como alóctonas, y confirma para la costa patagónica atlántica continental el aprovechamiento temprano de la obsidiana verde del seno Otway y gris verdosa veteada del cordón Baguales. Se concluye que el sitio arqueológico YQ 3 brinda nueva información respecto a la ocupación humana temprana entre el sur del río Santa Cruz y cabo Vírgenes, sumándose así al elenco de sitios que permiten la discusión del nicho ecológico de los grupos humanos que habitaron la costa atlántica patagónica durante el Holoceno medio.

PALABRAS CLAVE: cazadores-recolectores, registro arqueológico costero, Patagonia Meridional, Holoceno medio, arqueología neotropical

\section{YEGUA QUEMADA 3: A THE MIDDLE HOLOCENE ARCHAEOLOGICAL DEPOSIT IN MONTE LEÓN NATIONAL PARK (PATAGONIA, ARGENTINA)}

\section{ABSTRACT}

This paper presents the results obtained in the archaeological site Yegua Quemada 3 (YQ 3), Monte León National Park, Argentina. It is a stratigraphic deposit located one meter and ten centimeters deep on a cliff of eight meters high above the present coastline and is dated in the middle

a Dirección Regional Patagonia Norte. APN Vice Almirante O Connor 1188, San Carlos de Bariloche 8400. Rio Negro scaracotche@apn.gov.ar

b Laboratorio de Zooarqueología y Tafonomía de Zonas Áridas. IDACOR-CONICET/Universidad Nacional de Córdoba. Av. H. Yrigoyen 174, (5000) Córdoba.smunoz@conicet.gov.ar, patalobbia@gmail.com

c CONICET/FFyL-UBA/Instituto Nacional de Antropología y Pensamiento Latinoamericano. 3 de Febrero 1370/78, CABA danielasol.cm@gmail.com 
Holocene. Resources exploited include large and small mammals, birds, fish, and invertebrates, and involve a variety of techniques that allowed the use of different static, mobile, terrestrial and marine resources. The lithic material is made on local and allochtonous raw materials, and confirms the early utilization in continental Patagonian Atlantic coast of green obsidian from the Otway sea and banded greenish-grey from Baguales. In short, the archaeological site YQ 3 provides new information about early human occupation between the south of the Santa Cruz river and cape Virgenes and joins the cast of sites that allow discussion of ecological niche of human groups that inhabited the Patagonian Atlantic coast during middle Holocene.

KEY WORDS: hunter-gatherers, coastal record, Southern Patagonia, Midle Holocene, neotropical archaeology.

\section{INTRODUCCIÓN}

Los recursos costeros y marinos han sido importantes para las poblaciones humanas de Patagonia durante el Holoceno, como queda atestiguado por una diversidad de restos arqueológicos recuperados en distintos sectores de la región (Ej. Borrero, 2001; Cruz et al. 2010, 2015; Gómez Otero et al. 2015; Legoupil, 2003; Mansur, 2008; Moreno, 2008; Zubimendi et al. 2005). La información sobre el uso de este tipo de recursos está documentada desde muy temprano en Patagonia (Borrero, 1994-95; Castro et al. 2007; Salemme \& Miotti 2008), por lo que incluso se ha propuesto que las costas son sectores del espacio patagónico que podrían haber sido de particular importancia durante el poblamiento inicial de la región, constituyendo rutas litorales de desplazamiento (Miotti \& Salemme, 2004). Sin embargo, todavía no conocemos suficientemente cómo se organizaron estas ocupaciones costeras, ni con qué intervalos de tiempo y en qué contextos tuvieron lugar los movimientos que ellas involucraron. Tampoco sabemos si los humanos impactaron sobre las poblaciones animales locales, ni si éstos impactos potenciales pudieron tener consecuencias diferenciales para las poblaciones de invertebrados y vertebrados marinos así como para las poblaciones de vertebrados terrestres. En lo que respecta a estos últimos, el registro zooarqueológico de las costas de Patagonia Meridional en el Holoceno muestra gran variabilidad en la importancia que tuvieron los mamíferos terrestres y marinos a lo largo del tiempo (ver Muñoz, 2011). Esta variabilidad del registro arqueológico supera la dicotomía planteada por el registro etnográfico, que postula estrategias sustancialmente diferentes para el aprovechamiento de los recursos litorales, a saber cazadores recolectores marítimos y terrestres. En este trabajo se presenta la información arqueológica recientemente generada en el Parque Nacional Monte León (Provincia de Santa Cruz, Argentina) datada en el Holoceno medio y se discute la misma en relación con los datos existentes para entender el registro arqueológico temprano de la costa atlántica meridional así como las semejanzas y diferencias que este registro presenta respecto de los materiales conocidos para el Holoceno tardío.

\section{ANTECEDENTES Y OBJETIVOS}

El registro arqueológico de la costa atlántica continental de Patagonia es conspicuo para el bloque temporal definido por los últimos 3000 años radiocarbónicos (ver Castro et al. 2007: Cuadros 2 a 4). Sin embargo, cuando nos adentramos más allá de los 3000 años AP este registro resulta mucho más elusivo, estando caracterizado por espacios en los que los vacíos de información son importantes y los cuerpos de datos disponibles presentan resoluciones muy distintas. Las dataciones más antiguas conocidas para la costa de Santa Cruz provienen de la costa del Golfo San Jorge, en el sitio Cabo Tres Puntas, con una antigüedad de $6060 \pm 70$ años AP; los sitios Médano Alto, con $5790 \pm 80$ años AP y Médanos 1, con $6300 \pm$ 90 años AP. En la costa al sur del río Deseado se encuentra el Alero el Oriental con varias dataciones calibradas que se ubican entre los $6930 \pm 100$ y $5150 \pm 80$ y en la localidad arqueológica Punta Medanosa el sitio Punta Buque 3, con una edad calibrada de 5715-5600 años AP (Castro et al. 2007; Ambrústolo et al. 2011; Zilio, 2016). Al sur del río Santa Cruz, y dentro del actual Parque 


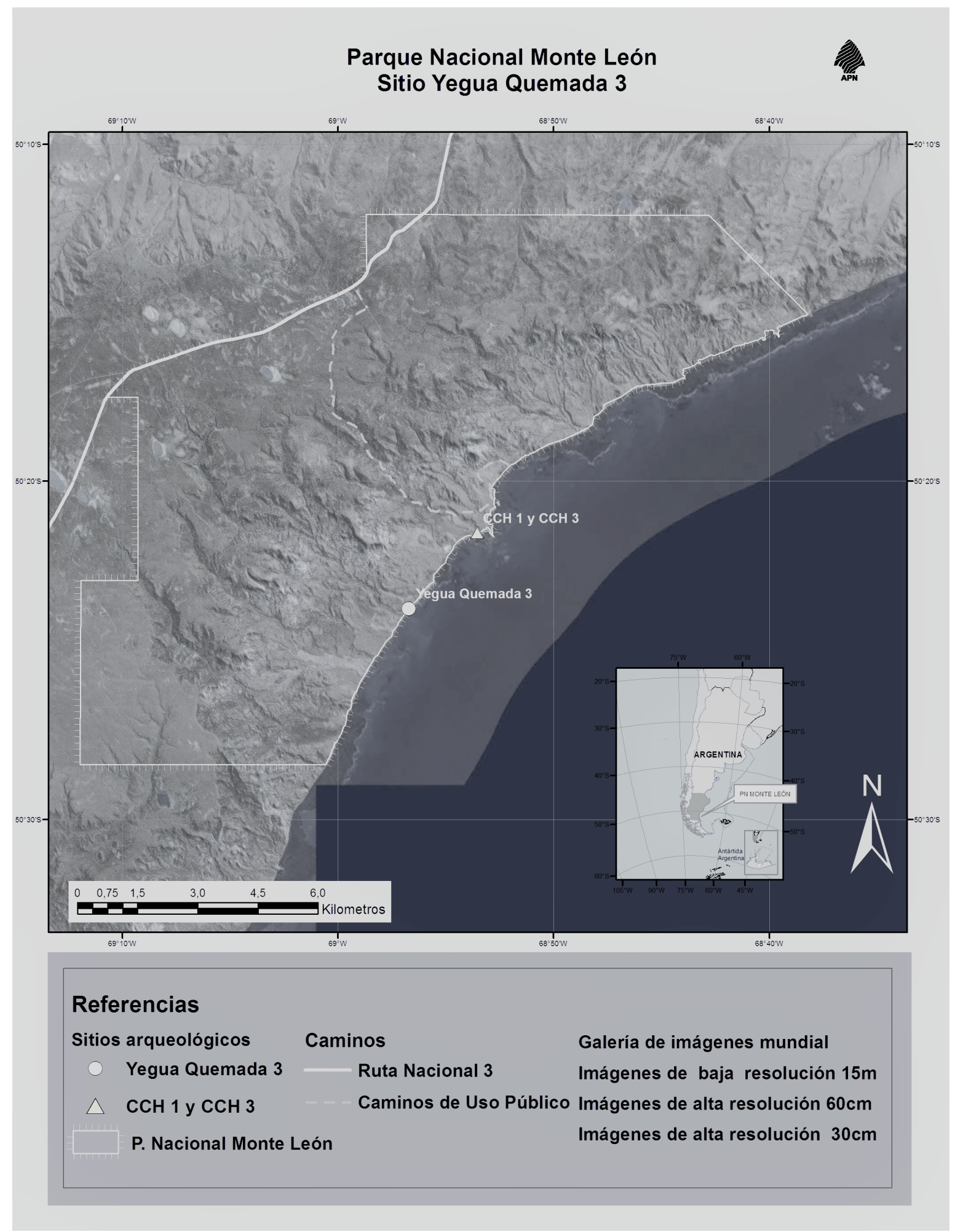

Fig. 1. Localización del sitio arqueológico YQ3 en el parque Nacional Monte León. 
Nacional Monte León (PNML), se han identificado por su parte los sitios CCH 1 (5550 \pm 90 años AP) y CCH 3 (4160 \pm 80 años AP) (Caracotche et al. 2005).

Con la excepción de la fecha procedente de Cabo Tres Puntas y CCH 1, que fueron tomadas sobre gasterópodos y bivalvos, respectivamente, las restantes fueron medidas a partir de restos de carbón, en depósitos compuestos principalmente por restos de moluscos con forma lenticular, asociados en algunos casos a materiales líticos, restos óseos y malacológicos. Se trata en general de registros-que muestran la profundidad temporal de la presencia humana en la costa atlántica meridional pero que no informan suficientemente sobre la ecología de los grupos humanos involucrados en estas ocupaciones. La excepción la constituye el ya señalado sitio El Oriental, ubicado en al sur del río Deseado, que ofrece una diversidad de clases de evidencia- en una estratigrafía de $30 \mathrm{~cm}-$, y que ha permitido discutir diversos aspectos relacionados con el uso pasado de los recursos en la costa del norte de Santa Cruz (Ambrústolo et al. 2011). Por ejemplo, su vinculación con una etapa de exploración inicial de la costa (sensu Borrero 1994-95) o con fuentes de aprovisionamiento de materia prima ubicadas a más de $300 \mathrm{~km}$ al oeste (Ambrústolo et al. 2011). Por su parte, en el sitio Punta Buque 3 ha sido identificado un enterratorio, el cual constituye el contexto de entierro más temprano conocido para la costa norte de Santa Cruz (Zilio, 2016).

En otras palabras, el registro del Holoceno medio tiene una representación dispar en la costa de la provincia de Santa Cruz (sur de Patagonia). En el sector norte existen claras evidencias de ocupaciones que hicieron uso de los recursos costeros desde el Holoceno medio (ca. 7000 años AP), las que han sido interpretadas como expresión de la ocupación inicial de estos ambientes. Las ocupaciones se hacen más claras, diversas y evidentes en el Holoceno tardío, tras un hiato de más de 3500 años, y tienen un alcance regional (Castro et al. 2007; Muñoz et al. 2009). En el sector meridional de la costa provincial, actual Parque Nacional Monte León, las evidencias son tenues en lo que respecta al Holoceno medio, ya que hasta el momento solo disponemos de estimaciones sobre la antigüedad de las ocupaciones, pero desconocemos las características y el rango de variabilidad de las mismas (Muñoz et al. 2009). En este trabajo se presenta nueva información generada a partir de los materiales recuperados en el sitio arqueológico Yegua Quemada 3. Se analiza el registro óseo de vertebrados $e$ invertebrados y el conjunto artefactual recuperado, y se discuten sus implicaciones en el marco recientemente reseñado.

\section{DEPÓSITO ARQUEOLÓGICO}

El sitio arqueológico YQ3 se encuentra ubicado en un médano compactado que forma parte de unos de los niveles aterrazados altos, respecto del nivel del mar, en la costa sur del Parque Nacional Monte León (Fig. 1). Se trata del relicto de una lente de conchero identificado a 1,10 m por debajo del suelo actual en una cicatriz de erosión expuesta en un frente acantilado (Fig. 2). El acantilado presenta una topografía descendente, con pendiente abrupta hacia la playa. Se encuentra cubierto en el sector superior por vegetación arbustiva y en la base presenta cantos rodados. La pendiente hacia la playa oscila entre $50^{\circ}$ y $60^{\circ}$ y la ladera presenta sectores de arena suelta y arbustos dispersos que retardaron en cierta medida- la intensidad de los fenómenos erosivos (Fig. 3). Tanto en las escorrentías, como a media ladera, se observan restos arqueológicos dispersos en superficie, producto de la circulación de agua que transporta materiales hacia la playa

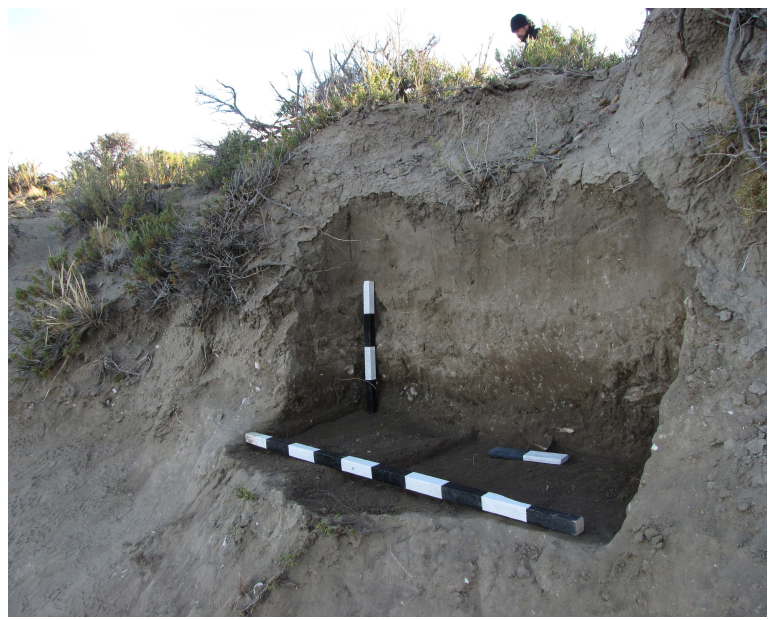

Fig. 2. Cuadrícula de excavación del depósito YQ 3. 


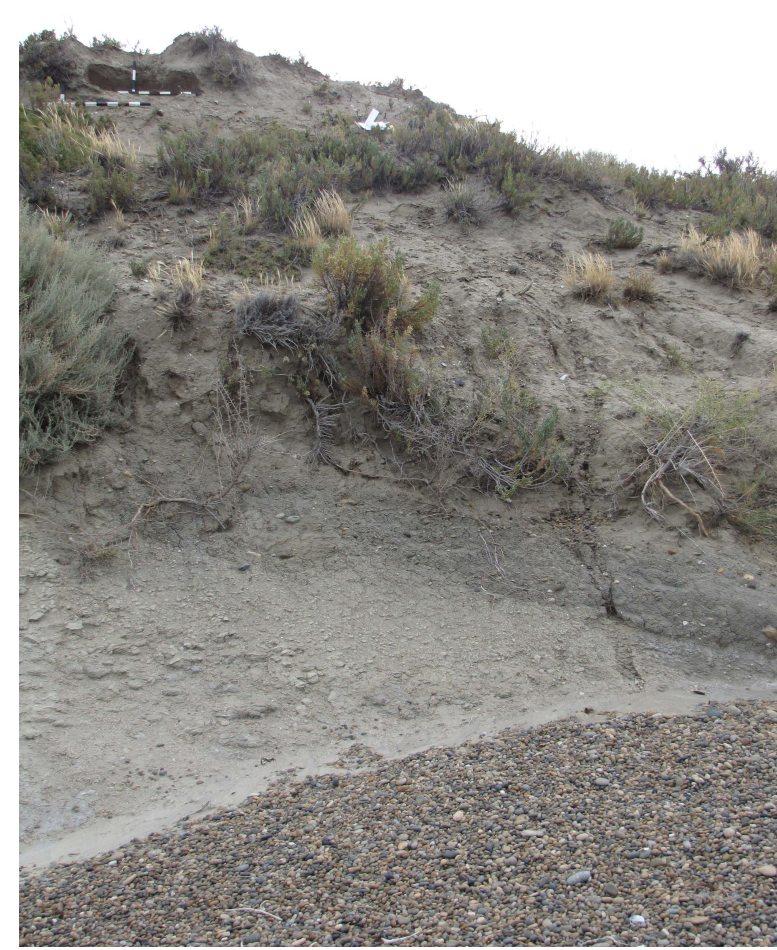

Fig. 3. Vista general de la ubicación del depósito.

de rodados, situación que marca que el depósito debe haber contado con una mayor extensión en el pasado (Fig. 3).

El depósito está ubicado a $8 \mathrm{~m}$ sobre la línea de costa actual del nivel del mar promedio $y$ se encuentra seccionado por un cañadón ubicado en cercanías del Cañadón de Yegua Quemada, que da nombre a la localidad (Figs. 2 y 3). El sustrato está compuesto por una matriz arenosa, con carbones de diferentes tamaños, raicillas y, en baja proporción, grava y gravillas. La estructura del depósito no presenta variaciones en cuanto a matriz, composición y tipo de procesos de formación observados entre los niveles excavados hasta una profundidad de $1,37 \mathrm{~m}$, nivel a partir del cual se registró un cambio de sedimento y una muy baja densidad de hallazgos (ver Resultados). Sin embargo, durante la excavación se observaron sectores cementados debido a la presencia de altos niveles de carbonato de calcio $\mathrm{CO} 3 \mathrm{CA}$ que precipitan desde el nivel 1 hasta el nivel 3 (B. Ercolano, com pers. 2009). Si bien la cementación observada fue mayor en el nivel 2 entre los 1,20 a $1,17 \mathrm{~m}$-, el carbonato de calcio se registró a lo largo de toda la estratigrafía hasta $1,30 \mathrm{~m}$ de profundidad, presentándose como concreciones adheridas a artefactos líticos, huesos y moluscos y en forma de pequeñas esferas dispersas desde el sector superior hasta los niveles inferiores del depósito. Una particularidad del depósito fue el hallazgo de pigmentos de ocre en muy bajas proporciones a $1,18 \mathrm{~m}$ de profundidad.

\section{METODOLOGÍA}

El relicto que permanecía en estratigrafía fue excavado por niveles artificiales a través de una cuadricula planteada de acuerdo a la forma que presentaba el médano. En su superficie máxima alcanzó a cubrir $1,25 \mathrm{~m}$ x 0,50 m, con una potencia de $30 \mathrm{~cm}$ (Fig. 2). El depósito arqueológico fue tratado como una sola unidad de análisis dado que los fechados obtenidos en los distintos niveles artificiales arrojaron dataciones similares correspondientes todas ellas al Holoceno medio (Tabla 1). El sedimento, que presentaba un $\mathrm{pH}=3,5$, medido a $1,10 \mathrm{~m}$ de profundidad, fue tamizado con una zaranda con malla de $2 \mathrm{~mm}$.

Los restos malacológicos fueron identificados al mayor nivel taxonómico posible siguiendo la nomenclatura de Aguirre y Farinati (1999); además se consultó la base de datos del Sistema de Información Taxonómica Integrado (ITIS, por su sigla en inglés). Para la cuantificación se tomó en cuenta el estado de conservación, clasificándose las valvas o conchas en enteras (VE) o fragmento identificable (FI). Se consideró valva o concha entera a todas aquellas que conservaban más del $90 \%$ de la pieza y que presentaban el elemento diagnóstico individual, es decir, el Elemento No Repetitivo (NRE) (Manson et al. 1998). Los fragmentos con menos del 90\% conservado pero que tenían presente el NRE fueron considerados como fragmentos identificables. La cuantificación se basó en el Número de Fragmentos Identificables (NISP) y el Número Mínimo de Individuos (MNI) para cada género o especie. En el caso de bivalvos el MNI tuvo en cuenta la lateralidad. La estacionalidad de muerte de los moluscos se analizó a partir de 
esclerocronología. Se seleccionaron 5 valvas de M. edulis en buen estado de conservación, con margen ventral sin fracturas ni desgaste en su borde. La preparación de las muestras se basó en el trabajo de Dunca y Mutvei (2001) y Schöne et al. (2005). Para la estacionalidad de muerte se siguió a Rhoads y Lutz (1980) y Kennish (1980).

Los restos de vertebrados fueron identificados a nivel taxonómico y anatómico (NSP, NISP y MNE). Para la identificación de las especies se utilizaron las colecciones de referencia del LaZTA y los manuales osteológicos de Murie (1870), Sanfelice y Ferigolo (2008), Pérez García (2003, 2008), en el caso de los pinnípedos, y Pacheco Torres y colaboradores (1986) y Sierpe (2015) para los camélidos. Los restos de aves fueron identificados a partir de la colección de referencia del IMHICIHU-CONICET y la guía de Gilbert y colaboradores (1996). Para la determinación de los restos de peces se consultó al Dr. Atilio F. Zangrando. Las modificaciones óseas incluyeron: meteorización, fracturas, daños por carnívoros, por pisoteo y marcas antrópicas, y fueron relevadas macroscópicamente por medio de lupas de mano de entre $3 \mathrm{x}$ y $15 \mathrm{x}$. Las variables consideradas para cada una de ellas fueron tomadas de la bibliografía (Behrensmeyer 1978; Lyman, 1994; Muñoz, 2008).

El análisis de los conjuntos líticos se basó en la propuesta de Aschero (1975, 1983), Aschero y Hocsman (2004) y Bellelli y colaboradores (1985). La determinación de la materia prima fue macroscópica con lupa de mano de 25x, a partir de la información sobre las rocas presentes en el área (Caracotche et al. 2008; Cruz et al. 2011; Fidalgo \& Riggi 1970) y observaciones personales de Cañete Mastrángelo. Se relevó estado de las piezas, es decir, si estaban enteras o fracturadas distinguiédose presencia o ausencia de talón en los desechos de talla (Bellelli et al. 1985). La cuantificación de los tamaños se hizo sobre piezas enteras y se consignó, además, en los desechos de talla el tipo de lasca (sensu Aschero 1975, 1983) a partir de piezas enteras y fracturadas con talón. Se observó la reserva de corteza en todas las clases tipológicas. En los instrumentos se analizó grupo tipológico, cantidad de filos y presencia o ausencia de filos complementarios.

\section{RESULTADOS}

Se recuperaron un total de 158 restos óseos y 206 restos malacológicos y cáscaras de huevo. 71 de los 158 restos óseos solo pudieron ser identificados como vertebrado indeterminado (35) y mamífero indeterminado (36). En buena medida esto se debe a que el conjunto está compuesto por restos de pequeño tamaño, siendo el $75 \%$ de ellos menores a los $4 \mathrm{~cm}$ de longitud. El conjunto artefactual incluye un total de 147 artefactos líticos, entre los cuales se encuentran dos núcleos, dos instrumentos, 142 desechos de talla y un percutor. Los fechados radiocarbónicos se realizaron sobre valvas de Aulacomya atra recuperados de la lente de moluscos a una profundidad de 1,12 m y sobre un hueso de Rhea pennata con huella de corte (AMS), recuperado en el nivel 2 a $1,13 \mathrm{~m}$ de profundidad. Ambas dataciones son asignables al Holoceno medio y se presentan en la Tabla 1.

Tabla 1. Dataciones radiocarbónicas efectuadas sobre materiales recuperados en YQ 3.

\begin{tabular}{lll}
\hline Material & $\begin{array}{l}\text { Edad } \\
\text { radiocarbónica } \\
\text { (años A.P.) }\end{array}$ & $\begin{array}{l}\text { Calibración por } \\
\text { efecto reservorio } \\
\text { (estimada) }\end{array}$ \\
\hline $\begin{array}{lll}\text { Valvas } \\
\text { (Aulacomya sp.) }\end{array}$ & $\begin{array}{l}6170 \pm 120 \text { (LP- } \\
2342)\end{array}$ & $5770 \pm 120$ \\
\hline Hueso Rhea sp. & $\begin{array}{l}5360 \pm 20 \\
\text { (UGAMS 11762) }\end{array}$ & - \\
\hline
\end{tabular}

\section{Conjunto Arqueomalacológico}

Los 206 especímenes recuperados fueron asignables a siete especies de los cuales 92,18\% corresponden a bivalvos y $7,82 \%$ a gasterópodos (Tabla 2). Entre los bivalvos se observa un predominio de la familia Mytilidae con tres especies Aulacomya atra, Brachidontes purpuratus y Mytilus edulis. En cuanto a la composición del sitio predominan A. atra $(46,96 \%)$, seguida por $M$. edulis $(24,35 \%)$ y B. purpuratus (20,87\%). Los gasterópodos, representados por cuatro especies, se hallan presentes en muy bajo número siendo Nacella magellanica la única considerada comestible.

Al determinar la estacionalidad de muerte por medio de técnicas esclerocronológicas no se encontró un claro predominio por una estación 
Tabla 2. Diversidad taxonómica en YQ 3. Conjunto de invertebrados.

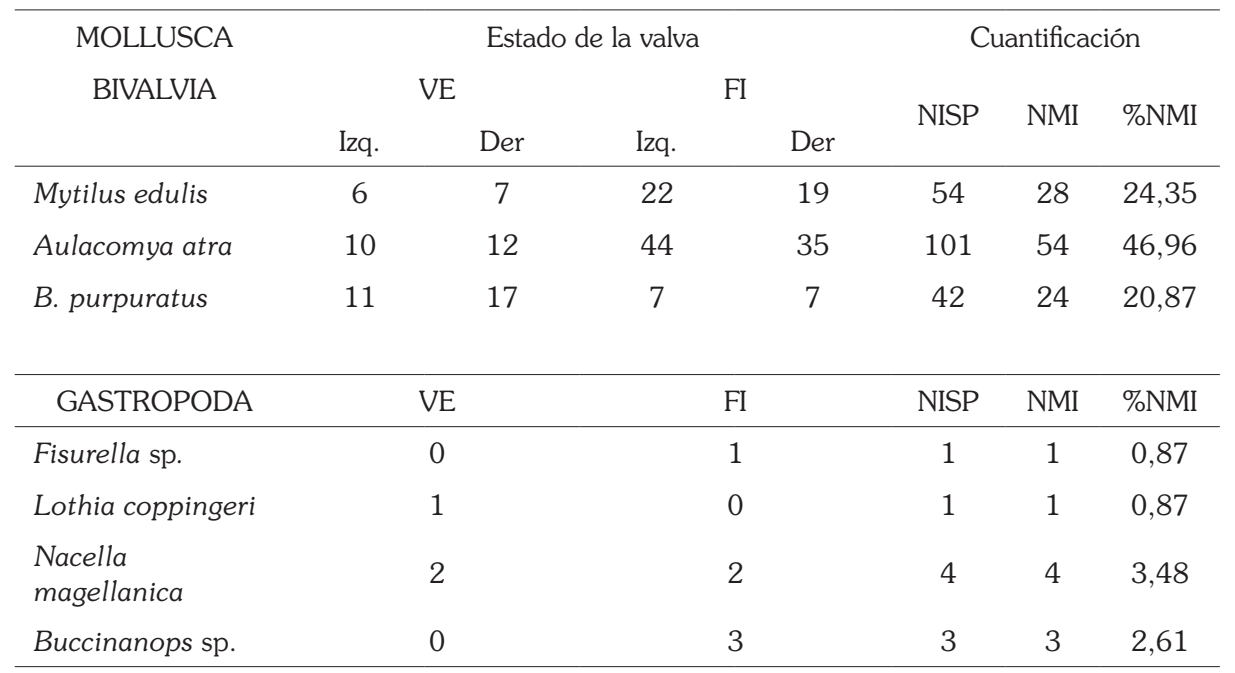

del año, aunque se destaca una mayor recolección centrada en verano (40\%) y otoño (40\%) y en menor medida en primavera (20\%), no obteniéndose registros asignables al invierno.

\section{Restos de Vertebrados}

La muestra presenta una diversidad importante en término de especies representadas, cubriendo el espectro de recursos faunísticos potencialmente utilizables en la zona (Tabla 3). Predominan los restos de mamíferos grandes
$(\mathrm{NISP}=55)$, seguidos por los de peces (NISP $=35)$, aves (NISP=22) y mamíferos pequeños (NISP=10). Si consideramos los tipos de animales involucrados encontramos que estas representaciones dan cuenta de animales terrestres (NISP $=38$ ) y marinos $(\mathrm{NISP}=49)$, grandes $(\mathrm{NISP}=26)$ y pequeños $(\mathrm{NISP}=61)$. En la tabla 3 se presentan estos datos con más detalle.

\section{Guanaco (L. guanicoe)}

Se recuperaron seis restos de guanaco que

Tabla 3: Diversidad taxonómica en YQ 3. Conjunto de vertebrados recuperado y proporción de especímenes con huellas antrópicas.

\begin{tabular}{lcccc}
\hline & NISP & NISP c/hu & $\%$ & MNE \\
\hline L. guanicoe & 6 & 2 & 33 & 6 \\
Pinnipedia & 14 & 1 & 7 & 10 \\
Rodentia & 6 & 0 & & 2 \\
$\quad$ Ctenomys sp. & 4 & 0 & & 3 \\
Aves & 16 & 2 & 13 & 7 \\
$\quad$ R. pennata & 6 & 2 & 33 & 4 \\
Pisces & 34 & 0 & & 33 \\
$\quad$ Merluccius sp. & 1 & 0 & & 1 \\
Vertebrados indeterminados (NSP) & 35 & & & \\
Mamífero indeterminado (NSP) & 36 & 2 & & \\
\hline Total & 158 & 9 & & 66 \\
\hline
\end{tabular}


corresponden principalmente al esqueleto axial. Se trata de una vértebra lumbar y otra torácica, prácticamente completas, y un fragmento de la cuarta vértebra cervical, este último con marcas de corte. Al esqueleto apendicular corresponden la epífisis distal de un metapodio sin fusionar, la tuberosidad del redondo mayor de un húmero izquierdo y una astilla ósea de un hueso largo no determinado. Con excepción del fragmento de húmero, que fue recuperado en la base del depósito, las restantes piezas se encontraron en el nivel superior del mismo y presentan estados de conservación diferentes, las vértebras lumbar y torácica con superficies óseas porosas y los restantes fragmentos con superficies óseas bien conservadas. Las vértebras torácica y lumbar y el fragmento de húmero presentan meteorización en estadio 3, aunque para la primera y el último éste proceso ha impactado de manera heterogénea ya que el primero presenta sectores en estadio 2 y el segundo en estadio 1 . Las marcas de raíces están presentes en el $67 \%$ del subconjunto, en restos con distinto grado de meteorización que fueron recuperados a distinta profundidad. En lo que respecta al número mínimo de individuos (MNI) los restos identificados corresponden a un individuo menor a cuatro años de edad, lo que fue determinado a partir del estado que presentan la vértebra lumbar y la epífisis de metapodio, que no alcanzaron la fusión ósea. Es decir, se trata de un individuo que aún no había alcanzado la plenitud reproductiva (Kaufmann, 2009). Las modificaciones de origen antrópico consisten en huellas de corte y están localizadas en el fragmento de vértebra cervical y el de húmero. El metapodio es el único espécimen con concreciones de carbonato en su superficie. $\mathrm{Si}$ bien no se identificaron fragmentos asignables a guanaco quemados, no se puede descartar que parte de los restos que presentan este tipo de daño, y que fueron identificados en la categoría Mamífero indeterminado, correspondan en realidad a esta especie.

\section{Pinnipedia}

Se identificaron 14 restos de pinnípedos que corresponden al esqueleto axial y apendicular. Se trata de un fragmento de cráneo, cuatro fragmentos de vértebras, uno de costilla, uno de escápula, otro de ulna, dos de fíbula y cuatro de metapodio respectivamente. El $21 \%$ de las superficies óseas se encuentra en estado fresco, es decir, sin meteorización. Un $43 \%$ de los materiales está levemente meteorizado (sensu Behrenmeyer, 1978 ) y el $14 \%$ restante alcanzó el estadio 3 . Dos especímenes presentan concreciones y en ninguno se registraron evidencias de quemado. Las marcas de raíces, por su parte, alcanzan al 50\% del total de restos recuperados $\mathrm{y}$, al igual que las concreciones de carbonato, afectaron a especímenes procedentes del nivel 2. La huella de corte se encuentra en la epífisis distal de la fíbula. La mayoría de estos restos no alcanzaron la fusión osteológica, correspondiendo a al menos dos individuos de distinta edad, un macho $O$. flavescens menor a doce años y otro mucho más pequeño de especie no determinada. Si se considera la representación del esqueleto axial y apendicular este conjunto muestra una composición dominada por la cabeza y el miembro anterior, seguida por el posterior y en tercer y cuarto lugar el esqueleto axial y las aletas

\section{Aves}

Los restos de aves suman un total de 22 especímenes, de los cuales seis corresponden a ñandú ( $R$. pennata), siete a aves voladoras no identificadas y nueve a aves indeterminadas. Los de ñandú son elementos del esqueleto axial: una primera costilla esternal completa semifusionada y una vértebra del sinsacro no fusionada y tres fragmentos del esqueleto apendicular, que consisten en huesos largos y una falange completa. La costilla y la falange presentan marcas de procesamiento, vinculadas con la desarticulación de la carcasa. Por otro lado se recuperaron fragmentos de cáscara de huevo que fueron analizados según la metodología propuesta por Apolinaire y Turnes (2010) para distinguir especie de Reídos. Estos autores consideran la cantidad de poros por centímetro cuadrado presentes en la superficie externa del huevo. Se escogieron dos fragmentos en buen estado de conservación y pudo determinarse que los mismos corresponden a $R$. pennata.

Los restos de aves voladoras consisten en cinco fragmentos de huesos largos, uno de ulna y otro de tiabiatarso. La ulna presenta huellas de machacado lo mismo que uno de los fragmentos de hueso largo que tiene este tipo de huellas y otras de corte. Los huesos 
de aves indeterminadas incluyen un fragmento de esternón, un escafolunar completo, un fragmento de radio y una vértebra cervical, además de cuatro fragmentos indeterminados y un fragmento de hueso largo. Estos materiales no presentan marcas de corte y solo uno de ellos está quemado.

Rodentia

El conjunto de roedores incluye 10 restos, cuatro de los cuales fueron identificados como Ctenomys sp. Estos últimos consisten en una vértebra sacra semifusionada, un fémur distal y un metapodio no fusionados. Los fragmentos identificados como roedores indeterminados incluyen dos fragmentos de huesos largos y cuatro fragmentos indeterminados, uno de los cuales está quemado.

Pisces

Los restos de peces consisten principalmente en cuerpos vertebrales $(\mathrm{NISP}=13)$, dos premaxilares, uno que corresponde a Merluccius sp. y otro que no pudo ser determinado, y 11 fragmentos indeterminados, uno de los cuales presencia rastros de combustión.

\section{Conjunto Artefactual}

Los 145 artefactos líticos incluyen dos núcleos y dos instrumentos, uno de dacita y otro de obsidiana verde en cada caso, 140 desechos de talla y un percutor de riolita (Tabla 4). Los núcleos están enteros pero presentan diferencias de tamaño. El de obsidiana mide $42 \times 20 \times 16 \mathrm{~mm}$, mientras que el de dacita mide $85 \times 75 \times 59 \mathrm{~mm}$. El número de piezas que están enteras o fracturadas pero que conservan su talón, suma 91 desechos. Si a este conjunto se agrega la obsidiana gris, cuya única pieza es un fragmento distal, se alcanza un total de 92 (Tabla 4, MNI desechos). Los desechos de talla corresponden a seis tipos de rocas, destacándose las silíceas y la dacita ( $\mathrm{N}=30$ y 28 respectivamente). Les siguen en importancia el grupo de las obsidianas, de diferentes fuentes como la verde de seno de otway ( $\mathrm{N}=17)$, que es la más abundante, la gris verdosa veteada proveniente del Cañadón de Baguales $(\mathrm{N}=4)$, la gris de Chaitén $(\mathrm{N}=1)$ y la negra de Pampa del Asador $(\mathrm{N}=1)$ y el basalto $(\mathrm{N}=11)$. Se han registrado también tres desechos de talla de calcedonia (Tabla 4). Los desechos de talla enteros son pocos, y de tamaños muy pequeño $(\mathrm{N}=8)$, pequeño $(\mathrm{N}=6)$ y grande $(\mathrm{N}=1)$. Estos incluyen seis tipos de lascas (Fig. 4), destacándose las angulares $(\mathrm{N}=66)$, seguidas por las planas $(\mathrm{N}=14)$, de dorso natural $(\mathrm{N}=6)$ y primarias $(\mathrm{N}=3)$. Finalmente, las menos representadas son las de arista y las secundarias ( $\mathrm{N}=1$ en cada caso). Estos resultados muestran una clara preponderancia de lascas internas por sobre las externas. En relación a esto, se ha observado que la amplia mayoría de los artefactos no presenta reserva de corteza, que

Tabla 4. Cantidad de piezas por clase tipológica y las materias primas identificadas dentro de cada una. Se discrimina el número mínimo para los desechos de talla. Obsidiana GVV: gris verdosa veteada, obsidiana G: gris, Obsidiana V: verde, obsidiana N: negra.

\begin{tabular}{lccccc}
\hline & \multicolumn{2}{c}{ DESECHOS } & INSTRUMENTOS & NÚCLEOS & PERCUTOR \\
\hline Basalto & $\mathrm{N}$ & MNI & MNI & MNI & MNI \\
Obsidiana GVV & 27 & 10 & & & \\
Obsidiana G & 4 & 4 & & 1 & \\
Obsidiana V & 1 & 1 & 1 & & \\
Obsidiana N & 24 & 16 & & & \\
Rocas silíceas & 1 & 1 & & & \\
Riolita & 40 & 27 & & & \\
Calcedonia & 3 & 3 & 1 & 2 & 1 \\
Dacita & 40 & 30 & 92 & & \\
\hline TOTAL & 140 & 92 & & & \\
\hline
\end{tabular}


suman 127 piezas, mientras que las piezas con reserva de corteza son 18 (16 desechos de talla, un núcleo y el percutor).

Los dos instrumentos consisten en un fragmento de filo no diferenciado manufacturado sobre obsidiana verde $y$ el otro es un denticulado frontolateral de dacita (Fig. 5). Éste último es el único que está entero y es de tamaño medianogrande. Ninguno de ellos posee reserva de corteza. Ambos instrumentos presentan ángulos mayores de $80^{\circ}$, lo cual significa que estaban embotados al momento de su descarte. Asimismo, los dos instrumentos sólo presentan un único filo por pieza.

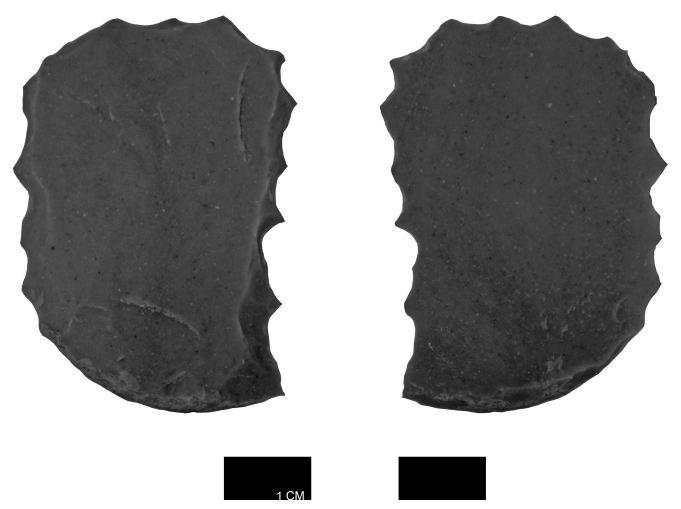

Fig. 5. Detalle del denticulado frontolateral.

\section{DISCUSIÓN}

El registro arqueológico recuperado en YQ 3 muestra una diversidad notable de materias primas líticas y una utilización de recursos marinos y terrestres de distinto tipo. En lo que hace a los recursos marinos, las especies representadas en YQ 3 muestran el uso de moluscos del intermareal y el sublitoral, con excepción de tres especies (un bivalvo y dos gasterópodos) que también son encontradas en el área supralitoral. Es decir predominan las especies cuyo hábitat se encuentra asociadas a fondos rocosos o de granulometría muy gruesa y aguas poco profundas de condiciones marinas semejantes a las actuales en las cercanías del sitio (Aguirre \& Farinati 1999; Zaixso, 2004). La explotación de estos invertebrados habría estado orientada hacia el aprovechamiento de los mitílidos (A. atra, M.

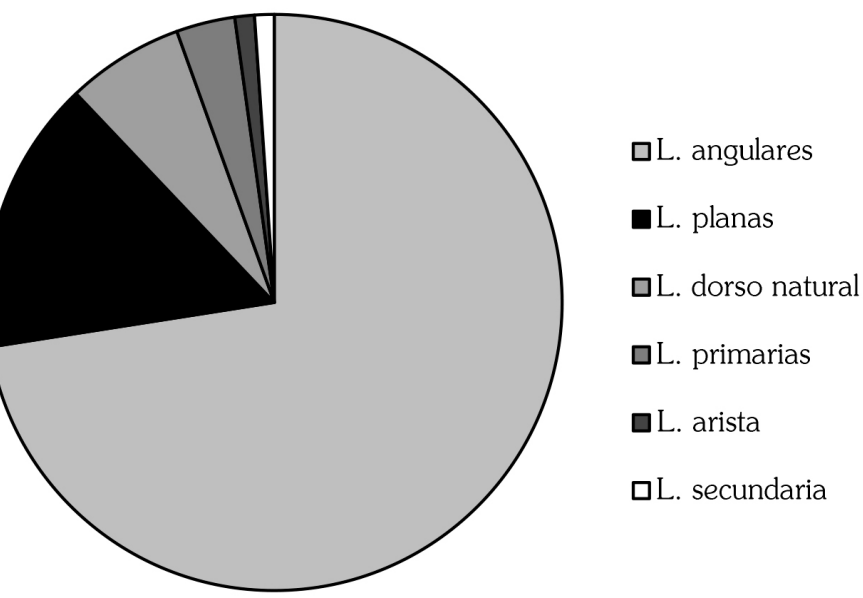

Fig. 4. Tipos de lascas representadas en el conjunto de los desechos de talla.

edulis y $B$. purpuratus) y centrada especialmente en $A$. atra que es la especie predominante. Los gasterópodos se hallaron en bajas proporciones y su única especie comestible, $N$. magallanica, se encuentra muy poco representada, lo que indicaría que no tuvo importancia significativa en la dieta. Esta composición defiere a lo encontrado en otros sitios más tardíos de la región Patagónica donde $M$. edulis constituye la especie predominante entre los bivalvos y N. magallanica entre los gasterópodos (Zubimendi \& Hammond, 2009; Franco et al. 2010; Zubimendi et al. 2011; Hammond \& Zubimendi, 2013; Hammond, 2014; Lobbia, 2016). La mayor recolección de determinadas especies en diferentes zonas del espacio costero patagónico puede ser indicativo de una distribución diferencial de especies debido a sus requerimientos ecológicos (i.e. diferencias de sustrato en distintas zonas costeras) más que a una selección al momento de la recolección. Entonces, si bien se puede identificar un patrón de selección de especies comestibles, la predominancia de una especie comestible frente a otra se da, en mayor medida, en virtud de la disponibilidad de las diferentes especies en los bancos de moluscos (Santiago, 2013). Estudios realizados por Zaixso (2004) sugieren que en los bancos de cholgas y bancos mixtos (cholgas y mejillones) se observa una dominancia de $A$. atra y en bancos de mejillones una dominancia de $M$. edulis, siendo A. atra poco accesible en estos últimos, ya que se desarrolla debajo de los mejillones y no quedan descubiertas durante la 
marea baja. Por lo tanto, la predominancia de A. atra en el sitio podría deberse a la presencia de bancos de cholgas o bancos mixtos en la zona de recolección.

Si bien los resultados sobre la estacionalidad de muerte de $M$. edulis no pueden considerarse determinantes, éstos sugieren que la recolección de estos invertebrados habría tenido lugar en las estaciones más cálidas (primavera verano otoño) sin que se pueda proponer un claro predominio de alguna estación en particular. Ests datos son parcialmente coincidentes con el registro de cáscaras de huevo de $R$. pennata, que sugiere primavera y principios de verano como estación de recolección de los mismos.

En lo que respecta a los pinnípedos, $O$. flavescens es una especie que habita actualmente la zona y es la que tiene una distribución continua en la costa atlántica. Los dos individuos representados en YQ 3 se ajustan a lo conocido para el registro arqueológico regional, en lo que a clase de edad y tamaño se refiere. En efecto, esta especie está representada en la mayoría de los registros arqueológicos informados para el Holoceno medio, en conjuntos en los que predominan los individuos adultos, aunque en algunos conjuntos también están representados individuos de distintas edades, incluso recién nacidos. No es posible señalar a partir de los escasos restos representados en YQ 3 si los individuos fueron obtenidos de manera aislada o en apostaderos, ya que los machos jóvenes son frecuentes en ambos tipos de situaciones. Sin embargo, si se los toma como parte de la diversidad taxonómica representada en el conjunto, es posible plantear que los lobos marinos habrían sido obtenidos a través de una estrategia oportunista que aprovechó el rango de diversidad disponible en la costa. En lo que hace a la representación anatómica los restos de otáridos corresponden a individuos que están representados de manera muy incompleta, respecto del número de carcasas fuente de las que proceden éstas porciones. Los miembros y el cráneo son las regiones anatómicas mejor representadas, mientras que los elementos axiales se encuentran presentes en una proporción menor, lo que demuestra una selección de partes semejante a la de conjuntos que han sido interpretados como resultado de captura de individuos aislados en otras zonas de Patagonia insular en el Holoceno tardío (ver por ejemplo Muñoz ,2005, fig. 2)

La presencia de peces en el depósito abre un interrogante sobre el modo de ingreso al registro arqueológico. Existen antecedentes de la presencia de distintas especies en el registro arqueológico regional, incluso de géneros como Merluccius sp. En un estudio reciente, Zangrando y Martinoli (2011) concluyen para la región del canal Beagle que durante los últimos 6000 años radiocarbónicos habría existido una distribución diferente de especies de aguas profundas, como Merluccius sp., que habría incluido aguas menos profundas. Es decir, Merluccius sp. podría haber estado más accesible durante gran parte del Holoceno, aunque no está claro qué tecnología se habría empleado en YQ 3 si éstos peces fueron obtenidos por medio de capturas directas. Una explicación alternativa es que los restos de peces hayan ingresado como parte de los contenidos estomacales de los pinnípedos. Esta vía de ingreso al registro arqueológico presenta complicaciones para su discusión ya que el grado de modificación de las partes duras de las presas consumidas por los pinnípedos está relacionada no sólo con el tipo de especie y el tamaño de los peces consumidos sino también con una multiplicidad de factores que intervienen azarosamente en cada comida (Tollit et al. 1997), lo que lleva a una gran variación intraespecífica en la importancia de los daños que sobre las partes duras consumidas pueden producir durante la digestión (Bowen, 2000). En términos generales, puede señalarse- sin embargo- que distintos estudios sugieren que los lobos y elefantes marinos poducen daños menos intensos que las focas (Bowen, 2000). En este sentido es importante señalar que Merluccius sp. forma parte de la dieta actual de O. flavescens, particularmente de los machos que tienen rango de acción más amplios que las hembras (Alonso et al. 2000). Por otro lado, si es que éstos restos no ingresaron como contenido estomacal, la estacionalidad obtenida a partir de cáscaras de huevo y esclerocronología resultaconcordante con la presencia de merluza en las aguas próximas, ya que la época en que frecuentan aguas someras es el verano (Zangrando, 2009, en Santiago 2013).

Las partes anatómicas de guanaco representadas en el conjunto corresponden mayoritariamente a sectores de rendimiento marginal 
y muestran un escaso procesamiento. En efecto, con excepción de la vértebra cervical y el fragmento de hueso largo, estos especímenes no fueron reducidos por medio de fracturas ni presentan marcas de procesamiento. Es decir, se trataría del descarte de partes de carcasas incompletas que pudieron ser transportadas junto con el equipamiento o, alternativamente, descartadas en este sitio mientras que las restantes piezas se transportaron a otro lugar. Algo similar ocurre con los restos de ñandú, que corresponden, a porciones marginales (como las falanges) y a porciones de mayor rendimiento como los huesos largos, las costillas o el sinsacro. Los restos de otros tipos de aves, representadas por partes anatómicas de rendimiento marginal (alas y patas), presentan huellas de procesamiento. En menor medida se determinaron fragmentos que corresponden a sectores de mayor rendimiento como el esternón.

El análisis del conjunto artefactual muestra que en YQ 3 se llevaron a cabo tareas de talla vinculadas principalmente a etapas intermedias, ya que los tipos de lascas más abundantes son los internas, es decir, sin corteza. Asimismo, existen evidencias del desarrollo de las primeras etapas de la reducción lítica, las cuales se ven reflejadas en la presencia, aunque escasa, de núcleos, lascas con corteza y un percutor. No se han registrado desechos de tamaño mediano y sólo se encontró uno de tamaño grande. Es posible que estos tamaños que resultan más apropiados para la confección de instrumentos, hayan sido usados y/o transportados a otros sectores del espacio. La mayor parte de las materias primas presentes en el conjunto son de origen local, lo cual muestra un conocimiento del área, en relación a la disponibilidad y accesibilidad (sensu Andrefsky, 1994) de las rocas aptas para la talla. Estas mismas rocas son las usadas en otros conjuntos del área correspondientes al Holoceno tardío. En YQ 3 se han recuperado sólo dos instrumentos, uno de los cuales es un fragmento de filo no diferenciado confeccionado sobre obsidiana verde y el otro es un denticulado de dacita que muestra una manufactura cuidadosa. Si bien los instrumentos son escasos para realizar inferencias acerca de la tecnología o de las posibles tareas que se realizaron con ellos, resultan muy informativos para conocer otros aspectos referidos a la variabilidad de la energía invertida en la talla lítica así como a la existencia de interacciones entre grupos y áreas a través de personas y/o ideas y/u objetos. La presencia de un instrumento y un núcleo de obsidiana verde junto a los desechos de talla de este mismo tipo y de las otras variedades cuyas fuentes fueron identificadas mediante el análisis geoquímico (Cruz et al. 2011, 2012), gris verdosa veteada, gris y negra- contribuyen a repensar estas ideas. Resulta llamativa la preponderancia de la obsidiana verde por sobre las demás, lo cual podría estar indicando mayor accesibilidad y/o una preferencia hacia este tipo, cuya fuente se localiza a mayor distancia que la de Cañadón de Baguales o Pampa del Asador. En este sentido, también destaca la presencia de obsidiana gris cuya fuente se encuentra localizada a más de $800 \mathrm{~km}$. Este escenario pone claramente en juego algún tipo de interacción a escala supraregional para comprender el uso de éstos espacios costeros en el Holoceno medio.

\section{CONCLUSIONES}

Diversos estudios han puesto en evidencia que las relaciones entre las poblaciones humanas y animales costeros han sido variables a lo largo del tiempo en Patagonia (Ej: Yesner, 1990; Legoupil, 1993-1994; Borrero, 1997; Yesner et al. 2003, entre otros). Como señala Borrero, esta diversidad de situaciones nos obliga a pensar en estrategias de investigación que trabajen sobre las señales sutiles de variación en el registro arqueológico, reconociendo que el espacio patagónico no es algo homogéneo, a pesar de que muchas veces ha sido tratado de esa manera (Borrero, 2001). Esta última consideración es particularmente importante para el estudio del uso de los hábitat costeros en el pasado, ya que el registro arqueológico muestra una variación apreciable, incluso en zonas donde éste es abundante, como en la costa norte de Santa Cruz durante el Holoceno tardío (Castro et al. 2003, 2007). Estos autores encuentran áreas que habrían sido utilizadas de manera continua y uniforme- como la del Golfo San Jorge- y otras que presentan escasas evidencias de uso- como la costa contigua entre Cabo Blanco y la ría Deseado- (Castro et al. 2007). Por el momento, para el Holoceno medio en la costa del Parque Nacional Monte León sólo disponemos de señales 
temporales de escasa resolución en términos del tipo de relaciones ecológicas que los grupos humanos plantearon con estos espacios y sus recursos. En este contexto, los datos generados a partir del estudio del registro arqueológico de YQ 3 muestran que en este sector de la costa patagónica se aprovecharon una diversidad de ambientes terrestres- $y$ marinos que involucraron la implementación de una diversidad notable de comportamientos vinculados a la captura de los animales- desde la simple cosecha de moluscos hasta la caza de animales móviles como el ñandú y el guanaco- y a la obtención de materias primas, que implican algún tipo de interacción a escala supraregional.

El registro arqueológico recuperado en $\mathrm{YQ}$ 3 es concordante con los datos conocidos para las ocupaciones costeras del Holoceno medio de la costa de Santa Cruz (ver Castro et al. 2007; Ambrústolo et al. 2011) y representa un aporte significativo para discutir la naturaleza de las mismas. Como se señaló, se trata del resultado de una ocupación sutil y acotada, que probablemente haya tenido lugar durante la primavera-verano, a juzgar por los indicadores de estacionalidad evaluados, y habría estado vinculada a un ambiente costero de condiciones similares a las actuales. Los resultados nos informan que se aprovechó la diversidad presente en este ambiente patagónico y que las presas capturadas fueron claramente procesadas, a juzgar por el pequeño tamaño de los fragmentos que componen la muestra y la presencia de huellas antrópicas vinculadas con el procesamiento en un $7 \%$ de los restos identificados. Todas las huellas fueron producidas por filos. Este aprovechamiento de la diversidad faunística indica un evidente conocimiento del ambiente costero ya para finales del Holoceno medio, que involucra vertebrados e invertebrados de distinto tamaño, pero también, y más importante aún, de animales que tienen comportamientos muy diferentes, abarcando desde recursos estáticos moluscos en distintos ambientes marinos- a los muy móviles como aves terrestres y voladoras que requieren técnicas de capturas muy variadas para obtener recursos con características tan disímiles. El análisis efectuado no sugiere, sin embargo, la implementación de una caza o recolección más selectiva como es claro más adelante en el tiempo, durante el Holoceno tardío. Esta diferencia podría deberse a una razón de funcionalidad, es decir, por el tipo de actividades representadas en el depósito, o podría responder a una propiedad intrínseca de las ocupaciones que caracterizan el Holoceno medio en este sector del espacio costero -superviviente a la transgresión marina-. En cualquier caso, las evidencias penecontemporáneas disponibles para la costa norte de Santa Cruz permiten postular que los grupos humanos que habitaron la zona habrían implementado estrategias planificadas en el marco de rangos de acción amplios, lo cual sugiere una ocupación efectiva de los espacios costeros (sensu Borrero, 1994-1995; Zubimendi, 2010). El análisis del sitio arqueológico YQ 3 es concordante con esta hipótesis ya que estaría relacionado con un sistema de uso del espacio basado en grupos pequeños, con una alta movilidad. Estos grupos formaban parte de poblaciones mayores, como muestran las evidencias de interacción a escala suprarregional, y habrían tenido un nicho ecológico amplio ya que estaban insertas en una comunidad ecológica caracterizada por una baja competencia (Muñoz \& Mondini, 2008).

\section{AGRADECIMIENTOS}

Este trabajo es resultado de los proyectos PIP/CONICET 112-201201-00359, UNPA 29/ A364 y SECyT UNC RES. 313/16. Autorización de investigación APN $\mathrm{N}^{\circ}$ 804. Agradecemos a todo el personal del Parque Nacional Monte León que nos asistió en la logística y tareas de campo. Los Guardaparques Mariana Martínez, Pablo Contreras, Cristian Vellido y los brigadistas Diego Brescia, Leonardo Martínez y Rubén Sosa junto a Gustavo Nauto colaboraron en las tareas de campo. Agradecemos a Fabiana Cantarell (SIBAPN) por su ayuda en la realización de la Fig. 1, a Pablo Fernández por habernos facilito bibliografía y a Lorena L'Heureux por facilitarnos el acceso a las colecciones del IMHICIHU.

\section{BIBLIOGRAFÍA}

Aguirre, M. L., \& Farinati, E. A. (1999). Taphonomic processes affecting late quaternary molluscs along the coastal area of Buenos Aires province, Argentina, Southwestern Atlantic. Paleogeography, Paleoclimatology, Paleoecology, 149, 283-304. 
Alonso, M. K., Crespo, E. A., Pedraza, S. N, García, N. A., \& Coscarella, M.A. (2000). Food habits of the South American sea lion, Otaria flavescens, off Patagonia, Argentina. Fish. Bull. 98, 250263.

Ambrústolo, P., Zubimendi, M., Ciampagna, M. L., \& Trola, V. (2011) Alero el oriental: evidencias de las primeras ocupaciones de la Costa Norte de Santa Cruz (Patagonia, Argentina). Revista Werkén, 14, 9-22.

Andrefsky, Jr. W. (1994). Material availability and the organization of technology. American Antiquity, 59, 21-34.

Apolinaire, E., \& Turnes, L. (2010). Diferenciación específica de Reídos a partir de fragmentos de cáscaras de huevos. Su aplicación en sitios arqueológicos del Holoceno Tardío. En M. Berón, L. Luna, M. Bonomo, C. Montalvo, C. Aranda \& M. Carrera Aizpitarte (Eds.), Mamül Mapu: pasado y presente desde la Arqueología Pampeana (pp. 215-222) Ayacucho: Libros del Espinillo.

Aschero, C. A. (1975) Ensayo para una clasificación morfológica de los artefactos líticos aplicada a estudios tipológicos comparativos. Informe al CONICET. Ms.

Aschero, C. A. (1983). Ensayo para una clasificación morfológica de los artefactos líticos aplicada a estudios tipológicos comparativos. Informe al CONICET. Revisión 1983. Ms.

Aschero, C., \& Hocsman, S. (2004). Revisando cuestiones tipológicas en torno a la clasificación de artefactos bifaciales. En D. Loponte, A. Acosta \& M. Ramos (Comps.), Temas de Arqueología. Análisis lítico (pp. 7-26). Buenos Aires: Talleres gráficos del Departamento de Publicaciones e Imprenta, dependiente de la Secretaría de Extensión Universitaria de la Universidad Nacional de Luján.

Behrensmeyer, A. K. (1978). Taphonomic and ecologic information from bone weathering. Paleobiology, 4(2), 150-162.

Bellelli, C., Guraieb, A. G., \& García, J. A. (1985). Propuesta para el análisis y procesamiento por computadora de desechos de talla lítica (DELCO- desechos líticos computarizados). Arqueología contemporánea, 2(1), 36-56.

Bowen, W. D. (2000). Reconstruction of pinniped diets: accounting for complete digestion of otoliths and cephalopod beaks. Can. J. Fish. Aquat. Sci,. 57, 898 905.

Borrero, L. A. (1994-1995). Arqueología de la Patagonia. Palimpsesto. Revista de Arqueología, 4, 9-69.

Borrero, L. A. (1997). The Origins of ethnographic subsistence patterns in Fuego-Patagonia. En C. McEwan, L. Borrero \& A. Prieto (Eds.), Patagonia, (pp. 60-81). Londres: British Museum Press.

Borrero, L. A. (2001). Cambios, continuidades, discontinuidades: discusiones sobre arqueología fuego-patagónica. En E. Berberian \& A. Nielsen (Eds.), Historia Argentina Prehispánica (pp. 815 838). Córdoba: Ediciones Brujas.

Caracotche, M. S., Cruz, Espinosa, I., S., Carballo, F., \& Belardi, J. B. (2005). Rescate arqueológico en el Parque Nacional Monte León (Santa Cruz, Argentina). Magallania, 33(2), 143-163

Caracotche, M. S., Carballo Marina, F., Belardi, J. B., Cruz, I., \& Espinosa, S. (2008). El registro arqueológico del Parque Nacional Monte León (Santa Cruz): Un enfoque desde la conservación. En I. Cruz. \& M. S. Caracotche (Eds.), Arqueología de la costa patagónica. Perspectivas para la conservación (pp. 147-159). Río Gallegos: Universidad Nacional de la Patagonia Austral - Subsecretaría de Cultura de la Provincia de Santa Cruz.

Castro, A., Moreno, J. E., Andolfo, M., Gimenez, R., Peña, C., Mazzitelli, L., \& Ambrústolo, P. (2003). Análisis distribucionales en la costa de Santa Cruz (Patagonia argentina): alcances y resultados. Magallania, 31, 6994

Castro, A., Moreno, J., Zubimendi, M., Blanca Videla, M. A., Ambrústolo, P., Mazzitelli, L. \& Bogan, S. (2007). Cronología de las ocupaciones humanas de la costa norte de Santa Cruz. En F. Morello, M. Martinic, A. Prieto \& G. Bahamonde (Eds), Arqueología de FuegoPatagonia. Levantando piedras, desenterrando hueso y develando arcano, (pp. 527-539). Punta Arenas: Ediciones CEQUA.

Cruz, I., Muñoz, A. S., \& Lobbia, P. A. (2010). Zooarqueología al sur del río Santa Cruz (Patagonia Argentina). Los restos de fauna de P 96 (Punta entrada) y CL 1 (P. N. Monte León). En J. R. Bárcena \& H. Chiavazza (Eds.), Arqueología Argentina en el Bicentenario de la Revolución de Mayo Tomo 1 (pp. 315-320). Mendoza: Facultad de Filosofía y Letras, UNCu e Instituto de Ciencias Humanas Sociales y Ambientales, Conicet.

Cruz, I., Caracotche, M. S., Stern, C., Muñoz, A. S., Suby, J. A., Lobbia, P. A., Ercolano, B., \& Cañete Mastrángelo, D. S. (2011). Obsidianas y otros indicadores de circulación y uso del espacio en Punta Entrada y P. N. Monte León (Santa Cruz, Argentina). En Libro de resúmenes VIII Jornadas de Arqueología de la Patagonia (pp 28-29). 
San Rafael: Museo de Historia Natural de San Rafael.

Cruz, I., Muñoz, A. S., \& Lobbia, P. A. (2011). La explotación de recursos marinos en la costa de Patagonia continental: Análisis de casos de Punta Entrada y Monte León (Santa Cruz, Patagonia Argentina). Revista de Estudios Marítimos y Sociales, 4(4), 31-41.

Cruz, I, Ercolano, B., Cañete Mastrángelo, D. S., \& Lemaire, C. R. (2015). P 96 (Punta Entrada, Santa Cruz): un sitio arqueológico costero al sur del río Santa Cruz. Relaciones de la Sociedad Argentina de Antropología, XL(1), 253-277.

Dunca, E., \& Mutvei, H. (2001). Comparison of microgrowth pattern in Margaritifera margaritifera shells from south and north Sweden. Am. Malacol. Bull., 16, 239 250.

Gómez Otero, J., Schuster, V., \& Svoboda, A. (2015). Fish and plants: The "hidden" resources in the archaeological record of the Northecentral Patagonian coast (Argentina). Quaternary International, 373, 72-81.

Fidalgo, F., \& Riggi, J. C. (1970). Consideraciones geomorfológicas y sedimentológicas sobre los rodados patagónicos. Revista de la Asociación Geológica Argentina, XXV(4), 430-443.

Franco, N- V., Zubimendi, M. A., Cardillo, M., \& Guarido A. L. (2010). Relevamiento arqueológico en cañadón de los mejillones (sur de la desembocadura del río santa cruz, argentina): primeros resultados. Magallania. 38(1), 269-280.

Gilbert, B. M., Martin, L. D., \& Savage, H. G. (1996). Avian Osteology. Missouri: Archaeological Society.

Hammond, H. (2014). Taphonomic analysis of archaeomalacological assemblages: shell middens on the northern coast of Santa Cruz (Patagonia, Argentina). Intersecciones en Antropología, 15, 2134.

Hammond, H., \& Zubimendi, M. A. (2013). Estudio de la composición de sitios concheros en la Costa Norte de Santa Cruz (Patagonia Argentina). En. F., Zangrando, R., Barberena, A. Gil, G. Neme, M. Giardina, L. Luna, C. Otaola, S. Paulides, L. Salgán, \& A. Tívoli (Eds.), Tendencias teórico metodológicas y casos de estudio en la Arqueología de la Patagonia (pp. 405-415). Buenos Aires: Altuna impresores.

Kaufmann, C. A. (2009). Estructura de Edad y Sexo en Lama guanicoe (Guanaco). Estudios actualísticos y arqueológicos en Pampa y Patagonia. Buenos Aires: Sociedad Argentina de Antropología.

Kennish, M. J. (1980). Shell microgrowth analysis: Mercenaria mercenaria (Linne') as a type example for research in population dynamics. En D.C. Rhoads \& R. A. Lutz (Eds.), Skeletal Growth of Aquatic Organisms (pp. 255 294). New York: Plenum Publishing Corp.

Legoupil, D. (1993-1994). El archipiélago del Cabo de Hornos y la costa sur de la isla Navarino: poblamiento y modelos economicos. Anales del Instituto de la Patagonia, Serie Ciencias Humanas, 22, 101-121.

Legoupil, D. (2003). Cazadores-recolectores de Ponsonby (Patagonia Austral) y su paleoambiente desde VI al III milenio A.C. Magallania Tirada Especial (Documentos), 31.

Lobbia, P. (2016). Análisis morfométrico en Mytilus edulis: una herramienta para el análisis de los sitios concheros y la arqueología de la costa. Revista Arqueología, 22, 141-165.

Lyman, R. L. (1994). Vertebrate taphonomy. New York: Academic Press.

Mansur, M. E. (2008) Arqueología de la zona de Punta Bustamante (Prov. de Santa Cruz, Argentina). En I. Cruz. \& M. S. Caracotche (Eds.), Arqueología de la costa patagónica. Perspectivas para la conservación (pp. 17-45). Río Gallegos: Universidad Nacional de la Patagonia Austral - Subsecretaría de Cultura de la Provincia de Santa Cruz.

Miotti, L., \& Salemme, M. (2004). Poblamiento, movilidad y territorios entre las sociedades cazadoras-recolectoras de Patagonia. Complutum, 15, 177-206.

Moreno, J. E. (2008) Arqueología y etnohistoria de la Costa Patagónica Central en el Holoceno Tardio. Rawson: Fondo Editorial Provincial, Secretaría de Cultura de Chubut.

Muñoz, A. S. (2005). Current perspectives on human-animal relationships in Atlantic Tierra del Fuego island, southern Patagonia. Before Farming, 2, 183-196

Muñoz, A. S. (2008). El procesamiento de los camélidos fueguinos en el pasado. Aspectos metodológicos y resultados alcanzados para el sector atlántico de Tierra del Fuego. En A. Acosta, D. Loponte \& L. Mucciolo (Comps.), Temas de arqueología. Estudios Zooarqueológicos y Tafonómicos (I) (pp. 77-97). Buenos Aires: Instituto Nacional de Antropología y Pensamiento Latinoamericano.

Muñoz, A. S. (2011). Human-pinniped relationships in Southern Patagonia. Current issues and future research agenda. En N. F. Bicho, J. A. Haws \& L. G. Davis (Eds.), Trekking the shore: changing coastlines and the antiquity of coastal settlement (pp. 305-332). Nueva York: Springer.

Muñoz, S., \& Mondini, M. (2008). Long term human/animal 
interactions and their implications for hunter-gatherer archaeology in South America.. En D. Papagianni, R. Layton \& H. D. G. Maschner (Eds.), Time and Change: Archaeological and Anthropological Perspectives on the Long Term (pp. 55-76). Oxford: Oxbow Books.

Muñoz A. S., Caracotche, M. S., \& Cruz, I. (2009). Cronología de la costa al sur del río Santa Cruz: nuevas dataciones radiocarbónicas en Punta Entrada y Parque Nacional Monte León (Provincia de Santa Cruz). Magallania, 37(1), 39-43.

Murie, J. (1870). Researches upon the anatomy of pinnipedia (part III). Proceedings of the Commitee of Science and Correspondence of the Zoological Society of London. Londres: Publications of the Zoological Society. Taylor and Francis.

Pacheco Torres, V. R., Altamirano Enciso, A., \& Guerra Porras, E. (1986). The Osteology of South American Camelids. Los Angeles: Institute of Archaeology, University of California.

Pérez García M. I. (2003). Osteología comparada del esqueleto poscraneo de dos géneros de Otariidae del Uruguay. Bol. Soc. Zool. Uruguay, 2(19), 1-16.

Pérez García, M. I. (2008). Ontogenia del postcráneo de Arctocephalus australis (MAMMALIA, Otaridae). Bol. Soc. Zool. Uruguay, 2(17), 1-19.

Rhoads, D. C., \& Lutz, R. A. (1980). Skeletal Growth of Aquatic Organisms. New York: Plenum Press.

Salemme, M. \& Miotti, L. (2008). Archeological hunter-gatherer landscapes since the Latest Pleistocene in FuegoPatagonia. Developments in Quaternary Sciences, 11, 437-483

Sanfelice, D., \& Ferigolo, J. (2008). Estudo comparativo entre os sincrânios de Otaria byronia e Arctocephalus australis (Pinnipedia, Otariidae). Iheringia, Sér. Zool., 98, 5-16.

Santiago, F. C. (2013). La ocupación humana del norte de Tierra del Fuego durante el Holoceno medio y tardio. Su vinculación con el paisaje. Ushuaia: Editora Cultural Tierra del Fuego.

Schöne, B. R., Dunca, E., Fiebig, J., \& Pfeiffer, M. (2005). Mutvei's solution: An ideal agent for resolving microgrowth structures of biogenic carbonates. Palaeogeography, Palaeoclimatology, Palaeoecology, 228, 149-166.

Sierpe, V. (2015). Atlas osteológico del guanaco (Lama Guanicoe). Chile: Ediciones Universidad de Magallanes.

Tollit, D. J., Steward, M. J., Thompson, P.M., Pierce, G. J., Santos, M. B., \& Hughes, S. (1997). Species and size differences in the digestion of otoliths and beaks: implications for estimates of pinniped diet composition. Can. J. Fish. Aquat. Sci., 54, 105-119.

Yesner, D. R. (1990). Fueguians and other hunter-gatherers of the subantartic region: 'cultural devolution' reconsidered. En B. Meehan \& N. White (eds), Huntergatherer demography: past and present. Sydney: University of Sydney: Oceania Monograph, 30, 1-22.

Yesner, D. R., Figuerero Torres, M. J., Guichón, R.A. \& Borrero, L.A. (2003). Stable isotope analysis of human bone and ethnohistoric subsistence patterns in Tierra del Fuego. Journal of Anthropological Archaeology, 22, 279291.

Zangrando, A. F. \& Martinoli, M. P. (2011). Fish ecology, conservation biology, and new insights from the archaeological evidence in the Beagle channel (Tierra del Fuego, Argentina). En S. P. Dempsey (Ed.), Fish Ecology (pp. 131-149). Nueva York: Nova Science Publishers, Inc.

Zaixso, H. (2004). Bancos de cholga Aulacomya atra atra (Molina) (Bivalvia: Mytilidae) del golfo San José (Chubut, Argentina): diversidad y relaciones con facies afines. Revista de Biología Marina y Oceanografía, $39(2), 61-78$

Zubimendi, M. A. (2010). Estrategias de uso del espacio por grupos cazadores recolectores en la Costa Norte de Santa Cruz y su interior inmediato. Tesis para optar por el título de Doctor en Ciencias Naturales. Facultad de Ciencias Naturales y Museo. La Plata: Universidad Nacional de La Plata. MS.

Zilio, L. (2016). Primer contexto mortuorio del Holoceno medio en la costa norte de Santa Cruz (Patagonia argentina). Magallania, 44(2), 219-224.

Zubimendi, M. A., \& Hammond, H. (2009). Análisis de los restos malacológicos en el sitio Los Albatros, Bahía del Oso Marino (Provincia de Santa Cruz). En M. Salemme, F. Santiago, M. Álvarez, E. Piana, M. Vázquez \& M. Mansur (Eds.), Arqueología de la Patagonia: una mirada desde el último confín (pp. 865-878). Ushuaia: Utopías.

Zubimendi, M. A., Castro, A S., \& Moreno, J. E. (2005). Procesos de ocupación de la costa norte de Santa Cruz: una síntesis. Relaciones de la Sociedad Argentina de Antropología, XXX, 225-233.

Zubimendi, M. A., Ambrústolo, P., Beretta, M., Mazzitelli, L., Hammond, H., Zilio, L., Ciampagna, L.,... Castro, A. (2011). Sitio Cueva del Negro: un caso de aprovechamiento intensivo de los recursos marinos en la Costa Norte de Santa Cruz (Patagonia, Argentina). Revista de Estudios Marítimos y Sociales, 4, 5162. 